\title{
RECONOCIMIENTO ARQUEOLÓGICO DEL BAJO ARAGÓN EN EL SIGLO XIX Y PRIMER TERCIO DEL XX: EVOCACIÓN DE SUS PROTAGONISTAS
}

\author{
ARCHAEOLOGICAL RECONNAISSANCE OF THE LOWER ARAGON \\ REGION IN THE LATE $19^{\text {TH }}$ AND EARLY $20^{\text {TH }}$ CENTURY
}

por

\author{
ENRIQUe VALLESPí PÉREZ
}

RESUMEN La comarca del Bajo Aragón, del valle medio del Ebro, destaca en la historiografía arqueológica española del primer tercio del siglo XX por su contribución al descubrimiento de las pinturas rupestres levantinas y a la sistematización de la prehistoria reciente y la cultura ibérica de su ámbito regional amplio. Un precursor singular, con sus anotaciones inéditas de campo, desde 1804 a 1832, y medio siglo después, desde 1897, un grupo de trabajo, el grupo generacional del "Boletín del Bajo Aragón", con propósitos comunes, actuaciones interrelacionadas e integración de sus excavaciones en las instituciones oficiales, hasta finalizado el primer tercio del siglo XX, son los protagonistas cuya evocación se pretende situar en una narración del proceso que pueda servir de sustento a los análisis.

ABSTRACT

Since the early XXth century, a rich tradition of archaeological research has produced a number of remarkable discoveries within the Lower Aragón region. This includes the discovery of Levantine rock art as well as the systematisation of the local Recent Prehistory and the Iberian culture. Between 1804 and 1832 there is a individual precursor of whom unpublished field notes are known. Half a century later, since 1897, the "Bajo Aragón Bulletin" generation began a series of excavations in co-ordination with official institutions that would last until the late 1920s. They are the main protagonists of the historiographic process leading to today's archaeology in the region.

Palabras claves Historiografía prehistórica. Arqueología. E. Colera Soldevilla, grupo del Boletín del Bajo Aragón. S. Vidiella, L. Pérez Temprado, M. Pallarés, J. Cabré, V. Bardavín.

Key words

Archaeology. Prehistoric Historiography. 
ENRIQUE VALLESPÍ PÉREZ

Sumario:

1) Primer tercio del siglo XIX: trabajos inéditos de E. Colera Soldevilla, desde 1804 a 1832. 2) Última década del siglo: comienzo de las actividades de S. Vidiella y L. Pérez Temprado. 3) Constitución de un grupo de trabajo: S. Vidiella, L. Pérez Temprado, M. Pallarés y J. Cabré (1897-1902). 4) Actividades en 1903 y 1904. 5) 1905 y 1906: actividades arqueológicas y preparación del "Boletín". 6) El "Boletín de Historia y Geografía del Bajo Aragón” y el reconocimiento de su aportación arqueológica (1907-1909). 7) Actividad arqueológica hasta 1914 e incorporación de V. Bardavíu. 8) Campañas del Institut d’Estudis Catalans con Bosch Gimpera y comienzos de la institucionalización de las investigaciones (1914-1922). 9) Excavaciones oficializadas y otras aportaciones. Óbitos de Pallarés, Vidiella y Bardavíu en la década de los veinte y últimos años de Cabré y Pérez Temprado.

\section{PRIMER TERCIO DEL SIGLO XIX: TRABAJOS INÉDITOS DE E. COLERA SOLDEVILLA, DESDE 1804 HASTA 1832}

Sin precedente significativo alguno y, por descontado, sin otro empeño de búsqueda intencionada, fue Evaristo Colera Soldevilla (Calaceite 1772-Valdealgorfa 1837) el precursor del reconocimiento arqueológico de la región. Cura rural, ilustrado y estudioso de su tierra natal, sus anotaciones y manuscritos originales sobre aspectos físicos, sociales y económicos y los arqueológicos pasaron tiempo después a su lejano pariente y continuador de su obra V. Bardavíu y fueron utilizados por S. Vidiella para trazas su biografía "Un Rector de Valdeltormo. Vida y obras del ilustre bajoaragonés D. Evaristo Colera Soldevilla", publicada en 1926, poco después de que $P$. Bosch Gimpera desvelara su interés historiográfico, afirmando, en 1923, que "en el siglo XIX los primeros trabajos sobre arqueología ibérica y finales de épocas anteriores fueron hechos en el Bajo Aragón por don Evaristo Colera Soldevilla. Desgraciadamente sus trabajos, que consignó en unas noticias manuscritas, quedaron inéditos. Merced a la amabilidad de don Vicente Bardavíu, su pariente, que los posee, he podido examinarlos"-en su encuentro con Bardavíu en Albalate del Arzobispo, en 1916-

Dedicado primeramente al estudio socioeconómico del país, Colera derivaría pronto hacia su pasado histórico, iniciando sus tareas arqueológicas de campo a comienzos del XIX, de cuya continuidad tenemos constancia desde 1804 hasta el año 1832, cinco años antes de su muerte y fecha del último de los manuscritos controlados. Desplegadas estas tareas arqueológicas desde sus dos parroquias de residencia, de Fabara y Valdeltormo, extendió Colera sus controles de noticias y comprobaciones directas de campo por el Bajo Aragón desde el tramo del Ebro entre sus afluentes Guadalope y Matarraña hasta las estribaciones del Maestrazgo, límite meridional de la comarca, en cuya actividad logró disponer de más de una docena de colaboradores locales, de distintos pueblos, a los que consideraba, en expresión pomposa, "amigos de la ilustración del país", sirviéndole de informadores y de enlaces en sus correrías de controles de vestigios y hallazgos, dejando en sus papeles observaciones, dibujos, descripciones y hasta informes detenidos, alguno de los cuales fue a parar a la Academia de la Historia, de una serie de poblados, sepulcros y hallazgos sueltos de la prehistoria reciente y protohistoria, y de ruinas y algún monumento romano y otros antiguos, de los términos municipales comarcanos controlados: de Fabara fue Colera valedor e introductor del conocimiento

1. Vidiella, en "Universidad. Revista de cultura y vida universitaria",de la Universidad de Zaragoza, con ejemplares en edición separada, Tip. La Académica, 1926; Bosch Gimpera, "Notes de Prehistòria aragonesa", en "Butlletí de la Associació Catalana d'Antropología, Etnología i Prehistòria", I, 1923:15-68 (la cita, en p.16). 
científico del sepulcro romano, a cuyo contexto local añadió los vestigios de cinco ruinas de construcciones en la Garita y otros restos en los Cinglos de la Muntfalla, además de alguna otra referencia en sitio innominado; de Valdeltormo anotó los poblados de las Torrazas, Más den Ríus y la Atalaya y las ruinas, en aceptable estado, de la Torre Quemada; de Mazaleón, el poblado de Mas de Escasos y sepulturas con ajuares en Tossal del Aguila y Val de Beltrán; del término municipal de Calaceite señaló las ruinas y vestigios del poblado de San Antonio, la extracción casual de dos tinajas de barro antiguas junto a la ermita de Santa Ana y la abundancia generalizada de fragmentos cerámicos en el término municipal, así como su concentración en una finca innominada de Cretas; entre Valdealgorfa y Torrecilla, los vestigios existentes en los Mases de Fraga; de Alcañiz, los poblados de Cabezo Palao y Alcañizel Viejo; y del Sur de la comarca, de Cantavieja anotó diversas antigüedades en un informe perdido; reunió además una nutrida colección de monedas ibéricas y romanas, con indicación de procedencia de términos y municipios de la comarca y del amplio marco regional, que quedaría también inicialmente en su legado inédito.

\section{2. ÚLTIMA DÉCADA DEL SIGLO: COMIENZO DE LAS ACTIVIDADES DE S. VIDIELLA Y DE L. PÉREZ TEMPRADO}

Inéditos, en su legado familiar, los manuscritos de Colera, ignoradas desde su muerte sus tareas y localizaciones arqueológicas y sin otra aportación alguna, el territorio comarcal permanecería vacío de evidencias arqueológicas conocidas, con excepción tan sólo del sepulcro romano de Fabara, trascendido su conocimiento con la publicación en el Boletín de la Academia de la Historia, en 1877, del viejo informe de Colera al P. La Huerta a comienzos del siglo y quizás de algún otro testimonio arqueológico inédito o inadvertido, como las anotaciones locales de mosén M. Valimaña en sus inéditos "Anales de Caspe" de 1842 y la exploración de Pablo Gil en Azaila y su posterior notificación por los hermanos A. y P. Gascón de Gotor en 1890. al tiempo que, en el último decenio del siglo, iniciaron sus actividades de campo S. Vidiella y L. Pérez Temprado, sin conocimiento mutuo, en sendos reconocimientos arqueológicos locales, de los términos de Calaceite y La Puebla de Hijar.

Santiago Vidiella Jasá, nacido en Calaceite (Teruel) en 1860, licenciado en Derecho en la Universidad de Zaragoza en 1881 y doctor, un año después, por la Universidad de Madrid, experto en derecho civil aragonés, presentó en su monografía "Recitaciones de la historia política y eclesiástica de Calaceite", en 1896, un capítulo inicial dedicado a sus reconocimientos del término municipal, que constituye la primera aportación de arqueología prehistórica de campo publicada de la región, con reseña de diversos hallazgos arqueológicos en los lugares de Valleta, Coll de les Moles, Fuente de Noró, Tosal Redó, Balsa del Coixet y Roca Caballera, Puch de San Antonio, Camino de Santa Ana en Racons y cercanías, en el término de Calaceite, y Castellans en el de Cretas, a cuya reseña añadió una "reflexión general del proceso de los periodos de la historia española" y la reflexión"concreta de su interpretación local", con sus "deducciones del examen de los restos".

Mientras, en esos mismos años de la actividad de Vidiella en Calaceite, iniciaba también Lorenzo Pérez Temprado sus reconocimientos arqueológicos en La Puebla de Híjar, de la misma comarca bajoaragonesa y provincia de Teruel; había nacido en Épila, provincia de Zaragoza, en 1865, hijo de maestro de primera enseñanza, y trabajó en el "Diario de Avisos" mientras cursaba la misma carrera en Zaragoza, trasladándose en 1890 a la Puebla de Híjar, donde tras dos años de docencia primaria, pasó a secretario del ayuntamiento, permaneciendo en La Puebla hasta su traslado a Mazaleón en 1897. Sería entonces, por indicación del erudito escritor alcañizano Francisco Zapater y Gómez, a quien recordaría años después, en publicación de 1907, como su "respetable amigo de grata memoria", cuando conoció la ubicación de la vieja intervención de 
Pablo Gil en el Cabezo de Alcalá de Azaila y fue en esos años de estancia en La Puebla, entre 1890 y 97 , cuando Pérez Temprado realizó el control arqueológico en su término municipal de restos de edificaciones prerromanas y romanas en Vasales, Ferradura, Paso de la Guardia, con hallazgos de monedas ibéricas y romanas; en Campo del Palacio, con parte de un mosaico bien conservado, cuya noticia daría a conocer escuetamente poco después de su traslado a Mazaleón, en artículo periodístico publicado en 1904.

\section{CONSTITUCIÓN DE UN GRUPO DE TRABAJO: S. VIDIELLA, L. PÉREZ TEMPRADO, M. PALLARÉS Y J. CABRÉ (1897-1902)}

Coincidiendo estas tareas personales, realizadas separadamente a finales del siglo, con el traslado de Pérez Temprado en 1897 a Mazaleón, la aproximación geográfica facilitó su encuentro personal con Vidiella y consiguiente relación y esta amistad recién establecida se vio pronto enriquecida, a comienzos del siglo, con la incorporación, en el mismo Calaceite, del joven Juan Cabré a las tareas arqueológicas locales, iniciada su vocación excavando con Vidiella el poblado de San Antonio en 1902, precisamente en el mismo año en el que Matías Pallarés se daba a conocer en este ambiente cultural de la región con la publicación en la prensa local de Alcañiz de su primer artículo de tema histórico bajoaragonés y entraba con ello en relación con los otros tres anteriores, cuya compenetración en sus propósitos queda reflejada en su correspondencia cruzada y en la serie de colaboraciones sobre temas históricos de la región, entre el citado año 1902 y 1904 , en la prensa local de Alcañiz, en "El Eco del Guadalope", de Pallarés, Vidiella y Pérez Temprado, y en las tareas arqueológicas de campo desplegadas localmente por éste y por Cabré. Fue precisamente el aumento creciente de todos estos trabajos, evidenciado en sus referidas entregas de 1904, y las limitaciones de la prensa local a sus propósitos, lo que impuso a sus autores la conveniencia de disponer de una publicación propia, cuyo proyecto se plantearon en ese mismo año y quedaría resuelto con la aparición, apenas dos años después, en enero-febrero 1907, de la primera entrega bimensual del "Boletín de Historia y Geografía del Bajo Aragón".

El despliegue cronológico detallado y la contemplación, en sus circunstancias personales, de esas tareas, realizadas entre 1897 y 1906 , evidencian la fuerza del empeño común que unía a sus cuatro protagonistas, cuya ilusión y compromiso de trabajo impregnan su correspondencia cruzada que he podido controlar.

Tales proyectos y quehaceres convergían en Santiago Vidiella, a la sombra de su dimensión universitaria y de la soltura de su propia posición personal. Nada sé de su dedicación desde que en 1896 publicara su monografía local de Calaceite hasta 1900, año en el que recibía, remitida desde Fabara el 1 de noviembre, carta con las copias de los signos lapidarios de los sillares del templo gótico parroquial y "el libro que trata del sepulcro romano, situado a corta distancia de la localidad y que Vds. desean conocer" (sin duda, el folleto publicado por Puig i Cadafalch y Brugués i Escuder ocho años antes), envío que había solicitado al secretario del ayuntamiento, Francisco Camón, conocido suyo; en 1902, unos meses después de la fundación de "El Noticiero" de Zaragoza, aparece Vidiella vinculado a ese periódico, así como al "Heraldo de Teruel" como "corresponsal en Calaceite y pueblos comarcanos" y en esa misma ciudad, con el Círculo de Recreo Turolense, siendo premiado en los Juegos Florales y por el Ateneo, en dicho año.

En Mazaleón, mientras tanto, Pérez Temprado, acomodado familiar y profesionalmente en su nuevo destino de secretario del ayuntamiento y ya en relación con Vidiella, iniciaba el reconocimiento arqueológico del término municipal, cuyos primeros controles notificaría poco después; muy sensible a las cuestiones de instrucción social, publicaba en "La Unión" de Teruel, del 23 de marzo de 1890, un soneto "A la memoria de D. Claudio Moyano", en la muerte de este "noble patricio", y en 1902 era premiado en los Juegos Florales 
de Zaragoza por un trabajo "Sobre el sello e historia de la villa de Mazaleón", muestra de la atención a su nueva circunstancia.

Iniciado el nuevo siglo con esta situación, a la relación establecida entre Vidiella y Pérez Temprado se unirían, en el mismo 1902, M. Pallarés desde Barcelona y J. Cabré en Calaceite, con lo que quedaría constituido su grupo de trabajo, que inició inmediatamente sus actividades: desde ese año y en los siguientes 1903 y 1904, la publicación en la prensa regional de Alcañiz de una nutrida serie de colaboraciones sobre temas bajoaragoneses; la intesificación de las actividades arqueológicas de campo; en el mismo año 1904, el desplazamiento de Pallarés desde Barcelona y su encuentro con Vidiella y Pérez Temprado, en excursión por los pueblos del Bajo Matarraña, iniciando con ello su dedicación compartida al estudio de la historia y la arqueología del Bajo Aragón; y el traslado en el mismo año de Cabré a Madrid, inicio de su lanzamiento nacional.

En esta conjunción de voluntades, fue Vidiella el canalizador de la unión de Pallarés y de Cabré, ambos desde sus diferentes circunstancias, a los afanes compartidos con Pérez Temprado.

Juan Cabré Aguiló (Calaceite 1882-Madrid 1949), el más joven de los cuatro, conocedor desde su infancia de las actividades de Vidiella en su ambiente común del Calaceite natal, había cursado, ingresando a los doce años, los dos primeros de carrera eclesiástica en Tortosa, en el 1894-95 y 95-96, y de vuelta a su pueblo, marchaba en 1899 a estudiar dibujo y pintura a Zaragoza e iniciaba en 1902 su relación estable con Vidiella y su vida arqueológica, excavando juntos, ese año, en el poblado de San Antonio de Calaceite, de modo que al ingresar en la Escuela de Bellas Artes de Madrid, en 1903 llevaba la inquietud arqueológica que movería sus primeros pasos en la capital de España, asumiendo y tramitando los proyectos comunes, conforme lo expondría poco después en cartas a Vidiella, que más adelante veremos.

Matías Pallarés Gil (Peñarroya, Teruel,1874-Barcelona,1924) estaba entonces en muy distinta circunstancia; trasladado muy pronto desde su pueblo natal a Barcelona, antes de saber leer (en carta familiar de recomendación se deseaba para él, además de trabajo, que al término de las jornadas pudiera, al menos, aprender a leer y escribir y los conocimientos básicos para poderse defender en la gran ciudad): trabajando en un taller de sastrería, autodidacto culto, sería, joven aún, el serio investigador de historia medieval y eficaz arqueólogo de los años posteriores, que veremos. En 1902 publicaba en la revista "Catalunya Artística" el primero de sus artículos de tema histórico bajoaragonés, cuya traducción reprodujo el "Heraldo de Alcañiz" y pasó a conocimiento de Vidiella, quien envió a Pallarés su monografía histórica de Calaceite publicada seis años antes, iniciándose de este modo su relación epistolar; las cartas que le envió Pallarés en este mismo año, en abril, julio y diciembre, rezuman su emoción del encuentro de ambas aficiones comunes por "las cosas de nuestra patria chica" e inician su intercambio de información.

\section{ACTIVIDADES EN 1903 Y 1904.}

En las primeras cartas que siguieron a su unión, la relación recién establecida entre los cuatro aparece convertida en la compenetración amistosa que les uniría definitivamente y que estimuló de inmediato el despliegue común de sus tareas.

La serie de aportaciones periodísticas a la historia regional se inició con la publicación, que hemos comentado, de Pallarés en la revista "Catalunya Artística" de 1902, de un artículo de tema bajoaragonés y su traducción en el "Heraldo de Alcañiz, que completó su autor con un segundo artículo en el mismo año y revista catalana, y con un artículo de Vidiella, de tema también tierrabajino, publicado al año siguiente en la revista "Aragón".

2. De M. Pallarés, "Una excursión por el Aragón catalán: santuario de la Virgen de la Fuente", trad. en "Heraldo de Alcañiz", 
en la intensificación de entregas durante el año 1904, cuya concentración en un mismo órgano de expresión, "El Eco del Guadalope" de Alcañiz, en su sección "Entrepáginas de historia y geografía regionales", abierta por iniciativa de Vidiella, y la regularidad de su aparición ponen bien a las claras el compromiso que impulsaba a sus autores, de cuyo acuerdo queda además constancia en su correspondencia epistolar de esas fechas.

Referidas todas ellas a temas de historia regional, Vidiella y Pallarés centrarían en las entregas una atención más exclusiva, mientras que Pérez Temprado las compaginaría con la actividad arqueológica de campo, que Cabré asumiría como dedicación exclusiva. La consideración actual de las entregas publicadas por Vidiella ${ }^{3}$, Pallarés ${ }^{4}$ y Pérez Temprado ${ }^{5}$ nos ilustran del cumplimiento dado por los tres implicados del grupo a su compromiso compartido de trabajo.

En estos mismos años, de 1902 a 1904, Cabré y Pérez Temprado desplegaban sus actividades arqueológicas.

Pérez Temprado, en su atención a la historia local, incluía en algunos de los artículos citados en "Entrepáginas" la noticia de sus antiguos controles, efectuados entre 1900 y 1907 en La Puebla de Híjar, a los que hemos hecho referencia, y sus recientes localizaciones de los túmulos de San Cristóbal, El Clot y Sol del Horta, con los que iniciaba su reconocimiento arqueológico del término municipal de Mazaleón.

Un Cabré muy joven desplegaba en esos años una doble actividad, iniciando en 1902 sus tareas arqueológicas locales en Calaceite y aledaños y empezando, con su traslado al año siguiente a Madrid, su lanzamiento nacional. En Calaceite realizaba, "casi sin descanso", "excavaciones por cuenta propia" más o menos cortas y "catas de mero tanteo" en los poblados dados a conocer años antes, en 1896, como hemos indicado, por Vidiella, principalmente San Antonio, Tosal Redó y Castellans y en otros por él controlados, Umbríes de Calaceite y en los colindantes pueblos de Cretas, Mas de Madalenes, y de Caseres, en la provincia de Tarragona, el Puig de la Gessera, cuyas tareas intermitentes, realizadas mientras cursaba en Zaragoza dibujo y pintura en la Escuela de Artes y Oficios y en el estudio de Mariano Oliver, como discípulo, se prolongarían varios años, quedando sin publicar hasta 1908 y 9. De este modo, cuando en 1903 se trasladaba a Madrid, iba Cabré, según confesión propia, con sus "aficiones al Arte y a la Historia", "a las manifestaciones de los tiempos prehistóricos", comentaba en otra ocasión, y consecuentemente, en ese mismo curso 1903-1904, de su ingreso en la Academia de San Fernando, entraba en relación con el P. Fita y le ponía al corriente de los hallazgos de Calaceite y entorno local, mostrándole además una selección de objetos representativos, cuyo interés trasmitió su valedor a la Academia de la Historia, con su informe y al parecer también el del director del Museo Arqueológico Nacional. En sus dos primeras cartas enviadas a Vidiella, el 12 de enero y el 7 de febrero, al poco tiempo de su llegada, Cabré le exponía entusiasmado el feliz resultado de las gestiones, hechas "en nombre de nuestra sociedad", recalcaba en su primera carta como expresión de la

1902, y "Excursions per l'Aragó catalá: mes noticies del santuari de la Mare de Deu de la Font", en "Catalunya Artística" del mismo año; y de S. Vidiella, "Los pergaminos de Mazaleón", revista "Aragón", 1903.

3. S. Vidiella, en "Entrepáginas", 1904: "De alcaides", "Capgrós o el bandolerismo bajoaragonés",'De la familia de Ariño", "Alcañiz, de la Orden de Calatrava", "Meteoros", "La autonomía de Alcorisa y Cretas", "Fabara y sus señores", "Indice razonado de los pergaminos de La Fresneda", "D. Francisco de La Torre y su Comedia Nueva", I y II.

4. M. Pallarés, en "Entrepáginas", 1904: "Un contrato matrimonial del año 1218. Primer hecho de armas de Jaime I el Conquistador", "La donación de Monroyo a los Calatravos. La carta-puebla de Peñarroya", "El tributo de cena y la cuestión que promovió en Monroyo", "Acto de recomendar la alcaldía y justiciado de Peñarroya", "De La Fresneda, I: la potestad real en los pueblos calatravos del Bajo Aragón", "Homenaje que prestó el comendador de La Fresneda y su nombramiento de lugar-teniente", "Colenda, Calanda, Foz-Calanda y Samper", "Albalate: su donación a la iglesia de Zaragoza. Notas sueltas", "De D. Francisco de Ariño", "Montalbán de la Orden de Santiago", "De los calatravos bajoaragoneses en tierras de Valencia", "De documentos del siglo XIV. Santuarios antiguos".

5. L. Pérez Temprado, en "Entrepáginas", 1904: "Maella, Azuara y Azuda”, "Sobre el sello e historia de la villa de Mazaleón", "Ilixis, Ixar Híjar", "Límites del obispado de Zaragoza", "Alcañiz en el año 1263", "La Puebla de Gaén o de Hijar", "De cómo se construyó y dotó la iglesia de La Puebla de Gaén hoy de Híjar", "Mazaleón y su ermita de San Cristóbal". 
solidaridad del grupo de amigos; se iniciaba así, tan tempranamente, la proyección a los ámbitos especializados de la incipiente arqueología prehistórica de la comarca bajoaragonesa.

Otro acontecimiento se sumaría a las intensas actividades desplegadas en el transcurso de ese mismo año 1904: el encuentro, en verano, de los tres amigos, Vidiella y Pérez Temprado, residentes en la comarca, con Pallarés, desplazado en tren desde Barcelona, acompañado "de un amigo de ésta", en excursión "fundidora de voluntades", a los pueblos del bajo Matarraña; de su encuentro en la estación de Fayón, el 31 de agosto, guardo la fotografía, obtenida sin duda por el acompañante, para mí anónimo, que se les unió "con el sólo objeto de participar en nuestra expansión y pasar unos días bajo la techumbre del cielo bajoaragonés", pero a quien hay que agradecer, además de su gesto, el testimonio gráfico de los tres amigos juntos. Durante su recorrido de diez u once días, desde Fayón a Nonaspe, Fabara y sepulcro romano, convento abandonado de La Trapa y Maella, los expedicionarios tuvieron tiempo para controlar los archivos municipales y parroquiales y anotar cuantos aspectos y edificios notables y noticias diversas de los lugares visitados consideraron de interés, así como también la existencia de vestigios antiguos en algún término municipal e incluso de camino desde Fabara a La Trapa no se les escapó el asentamiento en montículo de un pequeño poblado ibérico inédito; del intercambio de sus anotaciones queda testimonio en carta de Pérez Temprado a Vidiella el día 15 del mismo mes de septiembre, al poco del regreso. "respecto a los apuntes de nuestro viaje en aquellos que $\mathrm{Vd}$. no tomó, prontamente me ocuparé de ello y se los remitiré". Los resultados de este acopio de datos se vertieron, tres y cuatro años después, aunque sin referencias a la excursión, en varios artículos del "Boletín del Bajo Aragón".

\section{1905 Y 1906: ACTIVIDADES ARQUEOLÓGICAS Y PREPARACIÓN DEL "BOLETÍN"}

La continuidad en las tareas arqueológicas locales de Pérez Temprado y de Cabré, en Mazaleón y en Calaceite y Cretas, y los afanes de Vidiella, Pérez Temprado y Pallarés por disponer de una publicación propia, que encauzara y difundiera los estudios de la historia regional, polarizaron el trabajo de los componentes del grupo durante los dos años que siguieron al denso periodo que acabamos de contemplar. La aparición finalmente, en enero-febrero 1907, del "Boletín de Historia y Geografía del Bajo Aragón" iba a suponer el punto de inflexión de tales actividades.

Cabré, que armonizaba entonces, en los cursos 1904-5 y 1905-6, el segundo y tercer año de sus estudios de Bellas Artes en la Academia de Madrid con la continuación , en los veranos, de sus controles de campo de los poblados de Calaceite y Cretas (su "paisano y amigo", "el culto pintor calaceitano" "amoroso cultivador de las antigüedades comarcanas", le designaba Vidiella en sus publicaciones de esos mismos años) desveló en privado entonces su descubrimiento de las pinturas de la Roca dels Moros o dels Cuartos del barranco del Calapatá, cuya noticia había recibido, en 1903 ó 1905 de unos campesinos de Cretas y en el verano de 1906 "mostró a un grupo de personas entusiastas encargadas de divulgar el importante hallazgo para honra de la región", sin duda, el grupo de sus amigos, de los que sería Vidiella quien las diera a conocer en el "Boletín del Bajo Aragón"de marzo de 1907, ilustrado su texto con una lámina de las figuras de los dos ciervos afrontados de la composición principal, en dibujo, al parecer a mano alzada, de su descubridor.

Pérez Temprado proseguía su control arqueológico del término de Mazaleón, dedicado estos años al Piuró del Barranc Fondo y al reconocimiento de las cistas de San Cristóbal; en el primero, tras la recogida previa de algunos materiales por su descubridor y compañero de grupo, Julián Ejerique, con unas catas de comprobación; y en el barranco de San Cristóbal, con el reconocimiento de la dispersión de sepulturas y controles de urnas y algún material aflorantes y el comienzo del reconocimiento del poblado; pudo además retener para su estudio la lápida arábiga controlada el año anterior, que en noviembre de 1905 llevó a Calaceite 
y cuya transcripción averiguaría el amigo común Ejerique en Zaragoza, debida, según indican sendas cartas de Pérez Temprado a Vidiella, al arabista Ribera (publicada, dos años después, por el mismo Ejerique en el "Boletín del Bajo Aragón").

En cuanto a las publicaciones de historia regional, anteriormente expuestas, de Vidiella, Pallarés y Pérez Temprado en "Entrepáginas" de "El Eco del Guadalope", su incremento en 1904, con la extensión que iban adquiriendo algunos artículos, tropezaría pronto, desde finales de ese año, con las limitaciones inherentes a un periódico local en las circunstancias socioeconómicas de la época, lo que retrajo a los autores en sus entregas, limitadas, en lo que yo conozco de 1905, a tres artículos de Vidiella y una larga serie monográfica de Pallarés ${ }^{6}$, cuya extensión reafirmó a sus autores en la necesidad de contar con una publicación propia adecuada a sus fines, proyecto al que se entregaron desde entonces: Pallarés se decidió a rescatar de "Entrepáginas" y convertir en libro sus artículos monográficos sobre "La Caja de Valderrobles o Peña de Aznar la Gaya", imprimiéndolo en el mismo año 1905 y establecimiento tipográfico, E. Delgado, del periódico, y los tres amigos (recordémoslo, la tríada de las "Entrepáginas"), con un fluido intercambio epistolar y entrevistas de Vidiella y Pérez Temprado, se dedicaron al planteamiento y preparación de su deseada revista, concretándose su realización en el último trimestre del año: en octubre, Pérez Temprado exponía por carta a Pallarés su firme propósito de emprender de inmediato su realización y la respuesta, igualmente ansiosa y con aportación de sus ideas, la trasmitiría a un Vidiella que centraba y encauzaba desde siempre las ilusiones comunes; al mes siguiente, noviembre de 1906, ya estaban los tres amigos revisando sus trabajos para iniciar la publicación; sería director Vidiella y Pérez Temprado, redactor-administrador, se encargaría de los inminentes trámites de la edición. Apenas tres meses después, verían colmados sus afanes con la aparición, en enero-febrero de 1907, del primer número bimensual del "Boletín de Historia y Geografía del Bajo Aragón".

\section{EL "BOLETÍN DE HISTORIA Y GEOGRAFÍA DEL BAJO ARAGÓN" Y EL RECONOCIMIENTO DE SU APORTACIÓN ARQUEOLÓGICA (1907-1909)}

Un díptico volante de declaración de intenciones, apertura a colaboraciones y reclamo de facilidades y del concurso de ayuntamientos, párrocos, maestros y particulares "amantes del país", con el ruego de suscripciones (un año, España, 5 pesetas; extranjero, 7), fechado en 1 de febrero de 1907 y enviado nominalmente a los supuestos interesados, precedió la aparición, a fines del mismo mes, del primer cuaderno bimensual del Boletín. El compromiso adquirido (entregas en cuadernos bimensuales, formando cada año un tomo de 300 páginas) fue cumplido escrupulosamente, con todos sus cuadernos bien editados, durante los tres años que duró su publicación, hasta la entrega de noviembre-diciembre de 1909 en que cesó el Boletín, "porque a duras penas alcanza la suscripción a cubrir el coste material de impresión de números y esto le encierra en ámbitos estrechos, haciendo imposibles los desenvolvimientos y mejoras que acariciábamos", según la nota "Cesa esta publicación y por qué", que cierra su último cuaderno; conviene aclarar que no tuvo otra ayụda económica oficial que la suscripción de la Diputación de Teruel "a un ejemplar de la mencionada obra-las cinco pesetas anuales-, considerando-eso sí-que la publicación de que se trata merece toda clase de apoyo", remataba el oficio , de 3 de mayo de 1907, remitido al redactor-administrador de la revista, Pérez Temprado, a quien se autorizaba, con tal fin, a "revisar los archivos de los ayuntamientos de los pueblos de este provincia, por si en ellos encuentra algo utilizable para continuar la publicación". Hay que anotar

6. En "Entrepáginas" de 1905: de Vidiella, "D. Francisco de La Torre y su Comedia Nueva, III" (conclusión de su entrega anterior), "Un proceso célebre", "A la memoria de D. Salvador Pardo y Sastrón", y de Pallarés, la serie monográfica "La Caja de Valderrobles o Peña de Aznar la Gaya" 
también la concurrencia de otras circunstancias: la falta total de artículos, tanto de los habituales como de nuevos colaboradores, en los seis cuadernos bimensuales que componen el tercer y último tomo anual del Boletín, por una parte, y además, en el propio equipo de redacción, la intensificación de la actividad cultural de Pallarés con entidades e instituciones de Barcelona, donde vivía, y en la comarca, la entrega, prácticamente exclusiva desde entonces, de Pérez Temprado a las tareas arqueológicas, conforme luego veremos, con lo que, en estas circunstancias, tuvo que ser Vidiella quien afrontara con su mayor esfuerzo las sucesivas entregas del último año del Boletín.

Un "Plan de investigaciones", de la Dirección, en el tercer cuaderno del primer tomo, expone las materias a tratar: "1) Prehistoria, considerada como "la historia anterior a la reconquista, períodos prerromano, romano, gótico y arábigo"; 2) Reconquista y primeros tiempos que le siguieron; 3) Gobierno municipal; 4) Régimen económico administrativo del municipio; 5) Obras concejiles; 6) Ordinaciones o estatutos comunales; 7) Instituciones de enseñanza, beneficencia y religión; 8) Conquistas de autonomía municipal; 9) Moros, judíos, moriscos; 10) Tributos de los pueblos a la realeza; 11) Régimen feudal;12) Guerra de D. Juan II con los catalanes (1462-72); 13) Movimientos y guerra de Cataluña bajo D. Felipe IV (1635-52); 14) Guerra de Sucesión (1705-14); 15) Guerra de la Independencia (1808-14); 16) Hijos ilustres de las poblaciones; 17) Heráldica y genealogía; 18) Noticias meteorológicas, agrícolas y varias; y 19) Folk-lore".

El plan respondía con precisión a los materiales y áreas de estudio que llevaban entre manos los redactores, y su control y fecundo aprovechamiento de las fuentes locales y regionales, con algunas colaboraciones, especialmente la constante y destacada de Bernardo Gerona, amigo y componente del grupo desde sus tiempos de seminarista y luego de cura rural en la comarca, todo ello, enriquecido además con la ingente aportación de Pallarés desde el Archivo de la Corona de Aragón de Barcelona.

Vertebrada sobre esta contribución documental, la publicación del Boletín se completó con varias colaboraciones, algunas de componentes del grupo o con vinculación a sus tareas: colaboran en el tomo I, de 1907, Francisco Aznar Navarro, Dr. en Filosofía y Letras, desde Zaragoza; Julián Ejerique Ruiz, de Calaceite y componente del grupo; Joaquín Navarro Rebullida, maestro en Calaceite, amigo del grupo y autor también de los índices de los tres tomos, y Federico Pastor Lluis, de Tortosa; en el tomo II, de 1908, el citado J. Ejerique, Mariano Galindo, abogado, de Tortosa, Domingo Gascón Guimbao, abogado y publicista, cronista de la provincia de Teruel, desde Madrid, y el citado F. Pastor Lluis; y en el tomo III, de 1909, Gabriel Llabrés desde Santander". En el "Boletín" se reprodujeron además viejos textos de Casamayor, Zapater, Tablada y Valimaña y un artículo reciente del P. Furgús ${ }^{8}$.

7. Las aludidas colaboraciones al "Boletín de Historia y Geografía del Bajo Aragón" son las siguientes: de F. Aznar Navarro, "Ordenanzas de Andorra (siglo XVII)", "Relaciones concejiles. Albalate y Almochuel”, "Repoblación de Gea de Albarracín", en el t. I; de J. Ejerique Ruiz, "Dos bajoaragoneses ilustres", "De Maella", "Una inscripción arábiga”, "Villazgos de Castelserás", "Torrecilla y La Codoñera”, en el t. I, y “El célebre cura de Valdealgorfa don Ramón Segura”, en el t. II; de J. Navarro Rebullida, "Construcción de una iglesia", Pleitos entre Valderrobles y su aldea Torre del Compte", en el t. I y los índices de lugares y personas de los tres tomos; de F. Pastor Lluis, "Notas biográficas. El obispo Punter", en el t. I, y "El escudo de Morella: su historia y timbres (1233 a 1555)", en el t. II; de M. Galindo, "El R.P.M. Fray Jaime Jasá y Abás”, en el t. II y relación de publicaciones recibidas, en el t. I; de D. Gascón Gimbao, "Escritores de la provincia de Teruel", en el t. I y "Pedro Juan Zapater y su historia de Alcañiz", en el t. II; de G. Llabrés, "Más del escultor Forment y su familia", en el t. III.

8. Los textos recogidos en el "Boletín" de dichos viejos autores corresponden a sus siguientes obras: "Años políticos. 1782-1832", manuscrito inédito de Faustino Casamayor, "Historia de Alcañiz" de Pedro Juan Zapater, manuscrito inédito del año 1704 y "Mesa revuelta. Apuntes de Alcañiz" de Eduardo Jesús Tablada, de 1898, en el tomo II del Boletín y "Anales de Caspe" de Mosen Mariano Valimaña, manuscrito inédito del año 1842, en el tomo III; en cuyo tomo se recoge también el artículo de Julio Furgús, "Antigüedades ibéricas de Aragón", que había sido publicado en el mismo año en el "Boletín de la Sociedad Aragonesa de Ciencias Naturales". 
A los responsables del "Boletín" corresponde el grueso de los trabajos publicados, conforme evidencia el desglose individual de las aportaciones que se citan de Vidiella ${ }^{9}$, de Pallarés ${ }^{10}$ y de Pérez Temprado ${ }^{11}$, a los que se suma un artículo a Cabré ${ }^{12}$, además de una serie de noticias varias signadas por La Redacción ${ }^{13}$.

De contenido arqueológico, en el sumario reseñado, están los artículos de Vidiella, "Las pinturas rupestres del término de Cretas"y "Estaciones prehistóricas" de Calaceite, en el "Boletín" de 1907; de Cabré, "Hallazgos arqueológicos" de Calaceite y Cretas, "Boletín" de 1908; y de Furgús, "Antigüedades ibéricas de Aragón", también referido a Calaceite y Cretas, en "Boletín" de 1909; a lo que hay que añadir los datos de algunas otras referencias, esparcidos en varios artículos: Pérez Temprado, "Hijar: consideraciones sobre su antigüedad", con referencias arqueológicas a La Puebla de Hijar, en el "Boletín" de 1907; La Redacción, "Ejemplares de la escritura llamada ógmica o hemisférica", en Calaceite, "Boletín" de 1907; Vidiella, "Fabara","Boletín" de 1907; Valimaña, "Anales de Caspe" (inédito, 1842), en "Boletín" de 1909; Vidiella, "Calanda y FozCalanda", con referencia arqueológica a Castelserás, "Boletín" de 1909.

Tal aportación arqueológica, evidenciando toda la labor realizada desde antes por el grupo, tuvo una repercusión inmediata en las altas instancias especializadas, llamando la atención, en pleno curso de la publicación de los cuadernos bimensuales del "Boletín", de H. Breuil y del Institut d'Estudis Catalans, que acudieron a la comarca bajoaragonesa, a Cretas y Calaceite: Breuil, avisado desde Santander del descubrimiento de las pinturas por $\mathrm{H}$. Alcalde del Río, quien le envió un ejemplar del "Boletín del Bajo Aragón"correspondiente, y puesto en contacto con Vidiella ${ }^{14}$, se presentó en Calaceite en septiembre de 1908 y las estudió con Cabré en el barranco del Calapatá; y casi al mismo tiempo, se desplazaba desde Barcelona Josep Pijoan, enviado por el Institut, ante la publicación por Cabré de sus excavaciones en el poblado de San Antonio, en 1907 en el "Boletín de la Real Academia de Buenas Letras de Barcelona"y en el citado "Boletín del Bajo Aragón" al año siguiente, y a instancias de Pallarés, según el propio testimonio de Bosch, realizó una corta excavación en el poblado de San Antonio, en los primeros días de enero de 1909; las publicaciones de ambas intervenciones aparecieron sin demora, la de Pijoan llegó a incluirse en el "Anuari" de 1908, del Institut de Barcelona, y el estudio de Breuil y Cabré ,en "L'Anthropologie" de Paris, de 1909.

9. S. Vidiella, en el "Boletín": "Desarrollo del municipio de Alcañiz después de la Reconquista", "Las pinturas rupestres del término de Cretas", "La familia Forment", "Fayón y Nonaspe", "Fabara”, en el tomo I, 1907;"La Trapa de Maella", "La mujer de Forment", "Guerra de la Independencia", "Estaciones prehistóricas", "Unión y separación de Peñarroya y Fórnoles", en el tomo II, 1908; y en el III y último, de 1909, "Calanda y Foz-Calanda", "Don José Pardo y Sastrón", "Cosas de la tenencia de Valderrobles", "Estatutos municipales (Torredelcompte)" y "Contribución a la historia de Maella".

10. M. Pallarés, en el "Boletín": "La Hermandad de La Muela en Monroyo y Peñarroya", "Los seniores de Teruel", "El solar de los Entenzas en el Bajo Aragón", "Las fronteras sarracenas en tiempos de Berenguer IV", "Los señores de Peña de Aznar y Mazaleón", en el tomo I, 1907; "Don Pelegrín de Atrocillo, señor de Alcorisa", "La restauración aragonesa bajo Alfonso II el Casto", "Rectificación de un error", en el tomo II, 1908; y en el tomo III y último, "El infante primogénito don Jaime y su extraño enlace con Leonor de Castilla" y la conclusión de su artículo anterior "La restauración aragonesa bajo Alfonso II el Casto".

11. L. Pérez Temprado, en el "Boletín": "El retablo mayor de la iglesia de Fabara", "La expulsión de los llamados moriscos del Ducado de Híjar", "Hijar: consideraciones sobre su antigüedad. Documentos referentes a los primeros señores antes de là donación del rey Jaime I. Efemérides", "Reseña de la notable ermita de Mazaleón, en el tomo I, 1907; "De artistas y constructores, "Datos de la Guerra de la Independencia en los papeles de Mazaleón", "Heráldica municipal”, en el tomo II, 1908; y en el tomo III y último, de 1909, "De artistas y constructores (datos sueltos)".

12. J. Cabré, en el "Boletín" publicó "Hallazgos arqueológicos", en el tomo II, de 1908.

13. A La Redacción corresponden además una serie de noticias varias: en el t. I, "Datos sueltos", "Caspe: la puerta del Compromiso", "Valderrobles: iglesia arciprestal", "Ejemplos de la escritura llamada ógmica o hemisférica"; en el t. II, "Datos sueltos", "Heráldica municipal", "Los nombres de pagos y partidas como auxiliares de la investigación histórica"; y en el t. III y último, "Datos sueltos" y cerrando el tomo, la ya comentada nota "Cesa esta publicación y por qué". Hay también acuse de publicaciones recibidas, a veces reseñadas, de M(ariano) G(alindo) y de V(idiella), en los tres tomos.

14. Vicisitudes contadas por E. Ripoll Perelló, “El abate Henri Breuil (1877-1961)”, UNED, Madrid, 1994. 


\section{ACTIVIDAD ARQUEOLÓGICA HASTA 1914 E INCORPORACIÓN DE V. BARDAVÍU.}

Este reconocimiento, que coincidió casualmente con el cese de la publicación del "Boletín del Bajo Aragón", impulsaría las tareas arqueológicas del grupo, propiciando el lanzamiento nacional de Cabré, posibilitando el afianzamiento de la actividad de campo de Pérez Temprado en la comarca y alentando la plena dedicación arqueológica de Pallarés en Barcelona, donde actuaría de enlace de su grupo de amigos bajoaragoneses con Bosch Gimpera e incitaría su intervención con el Institut, cuyo comienzo en 1914 iba a coincidir con la incorporación arqueológica de V. Bardavíu, desde Albalate del Arzobispo. Los tres amigos, Cabré, Pérez Temprado y Pallarés y el recién incorporado Bardavíu, mantendrían desde entonces, de por vida, cada uno en sus propias circunstancias, su dedicación arqueológica, mientras que, en Calaceite, Vidiella, alma que había sido del Boletín, intensificaba sus estudios de historia regional y derecho aragonés, que completarían su obra. En esta perspectiva se sitúan las actuaciones personales de nuestros biografiados en el lustro transcurrido desde diciembre de 1909, final del Boletín, al comienzo de la intervención de Bosch Gimpera, en 1914, en la comarca.

Cabré, que acababa de publicar sus primeros trabajos arqueológicos de Calaceite y Cretas, seguía sus estudios pensionado en la Escuela de Bellas Artes de San Fernando en Madrid y colaboraba desde 1907 con el Marqués de Cerralbo en diversas de sus excavaciones, dejaría prontamente el ámbito local bajoaragonés e iniciaría su trayectoria nacional, hasta 1914 asociado al abate Breuil y desde ese mismo año, nombrado comisario de investigaciones de la recién fundada Comisión de Investigaciones Paleontológicas y Prehistóricas, desde los organismos arqueológicos centrales; en sus publicaciones de esos años están sus colaboraciones en 1913 y 14 con E. Hernández Pacheco y el Conde de la Vega del Sella; en ese último año estudiaba las pinturas, entonces descubiertas por C. Esteban, de la Val del Charco del Agua Amarga y completaba su estudio de los otros abrigos rocosos del Bajo Aragón, que incluiría en "El arte rupestre en España", en impresión avanzada y publicado un año después, en 1915, como núm.1 de las Memorias de la citada Comisión, con prólogo del Marqués de Cerralbo.

Pérez Temprado continuaba su control arqueológico del término municipal de Mazaleón y en estos años, de 1909 al 14, añadía a su repertorio los dos poblados contiguos de Escodines Altes y Baixes, en los que realizó catas en algunas viviendas y el control de una cista cercana, efectuaba también una corta excavación en el Piuró del Barranc Fondo, localizando una cista aislada cercana, y, en su control de la necrópolis del barranco de San Cristóbal, rescataba los fragmentos de cerámica pintada de la remoción no muy antigua de una de las cistas.

En estos años de reconocimiento del interés arqueológico de la comarca, la incorporación de Vicente Bardavíu Ponz (Alcañiz, 1866-Zaragoza, 1929), con su control inicial de yacimientos del término y entornos de Albalate del Arzobispo, desde poco después de 1907 hasta su publicación en 1914, iba a suponer, con la continuidad de su obra hasta 1926, la ampliación del campo de investigaciones a la zona occidental de la comarca. A su llegada a Albalate y toma de posesión de su parroquia, en el citado año 1907, tenía Bardavíu cuarenta y un años, llevaba dieciocho de sacerdote y se había licenciado en Filosofía y Letras en la Universidad de Zaragoza y doctorado en Teología en el Seminario Central de Toledo; en Zaragoza había ejercido la docencia como auxiliar en el Instituto de Segunda Enseñanza y desempeñado una cátedra de literatura del Seminario Pontificio, a cuya dedicación literaria corresponden sus publicaciones anteriores a las arqueológicas ${ }^{15}$. Dicha vocación arqueológica, que venía de sus estudios (declara haber tenido entre sus profesores a "mi queridísimo maestro D. Pablo Gil y Gil, explorador de las famosas ruinas de La Romana, entre Azaila y La Zaida"), está también relacionada con el legado de su pariente antepasado E. Colera

15. V. Bardavíu, "Apuntes de literatura general y española", Zaragoza, 1897, "Apuntes de literatura griega y latina", 1898, y "Fray Diego Murillo", 1901. 
Soldevilla, nuestro protagonista pionero en esta evocación historiográfica, cuyos manuscritos inéditos tuvo presentes Bardavíu en la elaboración de su primera publicación con aportación arqueológica, "Historia de la antiquísima villa de Albalate del Arzobispo", Zaragoza, 1914, en cuyo capítulo primero, "Prehistoria, Comienza la historia, Cartago y Roma", presenta su reconocimiento inicial del término y entornos locales de Albalate, con abundantes referencias arqueológicas, principalmente sepulcros y cuevas sepulcrales del Olivar de Macipe y Cueva del Subidor, en el Barranco de La Hoz, otras en el Barranco de la Val de Oria; una serie de poblados, principalmente Cantalobos, y restos romanos, además de una lista de yacimientos de la zona circundante y las habituales referencias literarias de la romanización de la región.; varias citas arqueológicas al "Boletín del Bajo Aragón", con alusiones a La Redacción, a Pérez Temprado y afectuosamente a Vidiella ("mi distinguido y cultísimo amigo", a quien cedería, años después, los manuscritos para la biografía de Colera) son testimoniales de la afinidad de sus propósitos con la obra del grupo.

De este modo, ausente Cabré, instalado en su ambiente nacional, desde Madrid, y mantenida la actividad arqueológica en la comarca, por la continuidad de Pérez Temprado en Mazaleón y su iniciación en Albalate por Bardavíu, los otros dos compañeros del grupo, sin Boletín entre las manos para sus densas entregas documentales, responderían también positivamente a las nuevas circunstancias, así, mientras en Calaceite Vidiella mantenía su plan de trabajo, entregado en estos años al estudio de los calatravos de Alcañiz, a recopilar temas y noticias varias de la historia regional e intensificar su atención al derecho aragonés, en Barcelona, su tierra de adopción, Pallarés, hasta entonces dedicado primordialmente a la historia regional del Bajo Aragón, se decidía en estos años por las tareas arqueológicas y su intervención sería decisiva para la actuación de Bosch en la comarca; perteneciente a diversas sociedades culturales de Barcelona, Club Montanyenc, Centre Excursionista de Catalunya y a la Unión Aragonesista, de la que fue presidente; de sus trabajos históricos anteriores, en 1909 aparecía "Don Blasco de Aragón, señor de Morella", en las Memorias del Congreso de Historia de la Corona de Aragón; en julio de 1911 era nombrado presidente de la Secció d'Arqueología, Arquitectura i Història del Club Montanyenc y en 1914 participaba en la fundación del Servei d'Investigacions Arqueològiques de l'Institut d'Estudis Catalans y, en afirmación del propio Bosch, sería Pallarés "quien indujo al Institut a emprender las excavaciones de Calaceite".

\section{CAMPAÑAS DEL INSTITUT D'ESTUDIS CATALANS CON BOSCH GIMPERA Y COMIENZOS DE LA INSTITUCIONALIZACIÓN DE LAS INVESTIGACIONES (1914-1922)}

Con la actuación de Bosch en el escenario del Bajo Aragón se iniciaría en 1914 un planteamiento continuado de las actividades arqueológicas de campo por los cauces institucionales: hasta 1920, con las campañas de Servei d’Investigacions Arqueològiques del Institut d’Estudis Catalans y desde entonces con las intervenciones de la Junta Superior de Excavaciones y Antigüiedades, sin solución de continuidad desde 1919 hasta cumplido el primer tercio del siglo, merced a las excavaciones en Azaila de Cabré y Pérez Temprado de 1919 a 1932 y las paralelas de este último desde 1924 en los términos de Fabara y Caspe (Zaragoza) y en el entorno provincial de la comarca, en el de Batea (Tarragoza).

En las campañas del Servei del Institut, desde 1914 a 1922, Bosch recapitularía inicialmente todo lo realizado con anterioridad por el grupo del Boletín, salvo las manifestaciones de arte rupestre, y emprendería un plan sistemático de excavaciones, personales del propio Bosch y bajo su dirección, por sus colaboradores J. Colominas y A. Durán i Sanpere en Calaceite y L. Pérez Temprado en Mazaleón, y los informes, publicados por Bosch en los Anuaris de 1913-14 y 1915-20, supondrían la incorporación a la literatura científica de la prehistoria reciente y protohistoria de la comarca, con sus manifestaciones eneolíticas, sus cistas y poblados prehistóricos e ibéricos. Realizadas las excavaciones en campañas anuales de verano, el calendario de actuaciones es buen reflejo de la actividad desarrollada: 
En 1914, Bosch excava totalmente La Gessera de Caseres, limítrofe, en la provincia de Tarragona, realiza una excavación de comprobación en el Tossal Redó de Calaceite y bajo su dirección, Pérez Temprado inicia en Les Escodines Baixes las excavaciones de los yacimientos del término de Mazaleón;

1915: en Calaceite, Bosch excava el Tossal Redó y en San Antonio y Colominas y Durán excavan el Vilallonc, mientras en Mazaleón excava Pérez Temprado Escodines Baixes y Escodines Altes, y desplazadoa Albalate del Arzobispo, en compañía de Colominas, Bosch revisa con Bardavíu sus localizaciones y materiales;

1916: Bosch en Calaceite prosigue las excavaciones del Tossal Redó y de San Antonio, al tiempo que Pérez Temprado sigue excavando Escodines Altes y empieza San Cristóbal y Piuró del Barranc Fondo; y ambos, en excursión a los yacimientos de la zona de Caspe, revisan La Tallada y Palermo, descubriendo en este último los fragmentos de la estela ibérica;

1917: Pérez Temprado sigue excavando San Cristóbal y El Piuró.

1918 y 1919: Bosch excava El Piuró, en Mazaleón, y San Antonio de Calaceite.

1920-22: Bosch excava en San Antonio, finalizando prácticamente con ello las campañas del Servei en la comarca.

Los resultados de este plan sistemático de excavaciones, con la garantía emanada de la dirección e intervención directa de Bosch Gimpera, venían a completar los tres lustros de trabajos de campo, descubrimientos, controles y catas de comprobación en los poblados, realizados y difundidos por el grupo del Boletín y que, a partir de la publicación de los informes del director de las campañas de excavaciones, quedaban convertidos en materia científica ${ }^{16}$. Bosch fue, por todo ello, el gran valedor del legado arqueológico del grupo generacional del "Boletín del Bajo Aragón".

Sobre estas campañas del Institut en el Bajo Aragón, cabe rememorar el interés recíproco que presidió su realización: Pijoan, tempranamente atento a las cerámicas ibéricas de Azaila y Calaceite, y Pallarés, valedor del grupo del Boletín ante Bosch y el Institut; Pérez Temprado, representante del grupo que tuvo la mayor relación de trabajo con Bosch, recordaría siempre la compenetración con la que esas campañas arqueológicas se desenvolvieron.

Al mismo tiempo y aparte de esas campañas de excavaciones dirigidas por Bosch, los componentes del grupo, incluido Pérez Temprado además de su participación en ellas, prosiguieron sus aportaciones novedosas.

Cabré, desde los organismos e instituciones centrales a los que pertenecía o con los que colaboraba, mantenía su atención a la comarca, publicando en 1914, con su descubridor C. Esteban, las pinturas del término municipal de Valdealgorfa, "La Val del Charco del Agua Amarga y sus estaciones de arte rupestre", separata anticipada de "El arte rupestre en España",de 1915, y en 1920, el sepulcro del Cañaret,"Un osario humano del eneolítico, de Calaceite, Teruel".

En Mazaleón Pérez Temprado, además de su colaboración con Bosch, descubría en 1918 las pinturas rupestres de Els Secans, publicadas con Cabré tres años después, y las de las Caídas del Salbime en 1920, el mismo año en el que aparecía la relación de sus localizaciones eneolíticas en covachos y campo abierto de La Horteta, Secans, Sol de Huerta y Era de Rayos, en artículo de Bosch en el Anuari recién publicado.

Vidiella publicaba en 1918, en la "Revista de Derecho Privado", de Madrid, "De la legítima de descendientes en Aragón", y en 1920 difundía en "La Zuda", revista del Ateneo de Tortosa, "Las antigüedades ibéricas de Calaceite" y desplazándose, en fecha que no puedo precisar, desde Calaceite a la zona ribereña del Ebro, de la desembocadura del Guadalope, comprobaba el interés arqueológico de los poblados de La Tallada y Palermo, en el término municipal de Caspe, anotados por Valimaña en sus manuscritos inéditos de 1842, y descubría la estela ibérica empotrada en la ermita de San Marcos, de Chiprana.

16. P. Bosch Gimpera, "Campanya arqueológica de l’Institut d’Estudis Catalans al limit de Catalunya i Aragó: Caseres, Calaceit i Maçalió", "El sepulcre del Canyaret a Calaceit", "Consideracions generals sobre les estacions eneolítiques del Baix Aragó i del Regne de Valencia", "Les investigacions de la cultura ibérica al Baix Aragó", en los Anuaris V, 1913-14, y VI, 1915-20, como referencias iniciales de su brillante bibliografía de síntesis posterior. 
En la zona occidental de la comarca, que había incorporado a estas tareas con la publicación en 1914 de su monografía local de Albalate del Arzobispo, Bardavíu iniciaba el estudio de los yacimientos entonces controlados y en 1918 publicaba a sus expensas "Estaciones prehistóricas y poblados desiertos, recientemente descubiertos y estudiados en la provincia de Teruel", de entre los ríos Martín y Guadalope; la intensificación, unos años después, de sus trabajos de campo y publicaciones afianzaría la integración de esta zona al núcleo arqueológico comarcal.

Fuera de su tierra natal, en su Barcelona adoptiva, Pallarés, tras su afortunada intervención ante Bosch y el Institut para la realización de las campañas en el Bajo Aragón, y definitivamente centrado en lo arqueológico, como hemos indicado, participó personalmente en el Bajo Aragón, con varias excursiones de las campañas del Institut y recién incorporado al personal de Servei como "comisionat d'excavacions", emprendía en ese mismo año 1915 sus tareas arqueológicas, de localizaciones y controles de yacimientos y principalmente, una serie continuada de excavaciones en diversas comarcas catalanas y en la provincia de Castellón, mantenidas desde entonces y que en 1920 presentaba la siguiente aportación de cuevas y monumentos megalíticos excavados y en su mayor parte publicados: cuevas de San Julià de Ramis (Cova y Cau de Les Goges), Cova de Joan d’Os de Tartareu, Cau Pasqual en Castelví de la Marca, Cova Fonda de Salamó, Cova de Solanes y, en Castellón, las cuevas de La Valltorta, y del mundo megalítico catalán, el sepulcro de Santa Mariá del Corcó y los megalitos del Bajo Ampurdán; sus informes, dando cuenta de estas excavaciones, se publicaron todos en el Anuari VI, 1915-1920: "El solutrià de Sant Julià de Ramis: el Cau de les Goges", "Exploració dels jaciments prehistòrics de la Valltorta", "Els sepulcres megalítics del Baix Empordá" y "Galería coverta de Puig-ses-Pedres (Santa María del Corcó)". Fiel además a su trayectoria investigadora de la historia medieval de Aragón, Pallarés publicaba en 1917 una conferencia pronunciada en el Centro Aragonés de Barcelona, en febrero de ese año, sobre "Aragón y el Fuero de Sobrarbe"17.

\section{EXCAVACIONES OFICIALIZADAS Y OTRAS APORTACIONES. ÓBITOS DE PALLARÉS, VIDIELLA Y BARDA VÍU EN LA DÉCADA DE LOS VEINTE Y ÚLTIMOS AÑOS DE CABRÉ Y PÉREZ TEMPRADO}

La década de los años veinte cierra prácticamente la contribución del grupo bajoaragonés a la arqueología e historia de la comarca, con una normalización de las excavaciones arqueológicas a través de instituciones adecuadas: con la Junta Superior de Excavaciones y Antigüedades, Cabré y Pérez Temprado, codirigiendo las excavaciones de Azaila y el segundo, además con excavaciones personales en Fabara, Caspe y Chiprana (Zaragoza) y Batea (Tarragona), y Bardavíu, asociado a P. Paris, con L École des Hautes Études Hispaniques, de Burdeos, en 1924 en poblados de Alcañiz. Pallarés, por su parte, seguiría en estos años sus excavaciones en Catáluña, de yacimientos en las provincias de Barcelona y Tarragona, con las campañas del Institut, de cuyo Servei d Investigacions Arqueològiques era, como hemos visto, activo componente desde su misma fundación.

Los fallecimientos, en esta década, de Pallarés, Vidiella y Bardavíu entre los años 1924 y 29 y, al comienzo de la década siguiente, el cese en su actividad arqueológica de Pérez Temprado, con su jubilación profesional en 1932, y el bloqueo que las circunstancias nacionales impusieron a la continuidad de Cabré en Azaila, sitúan al final de este primer tercio del siglo la conclusión conjunta de las aportaciones de los cinco protagonistas; después, en sus últimos años, Cabré, con su corta reanudación de la excavación de Azaila hasta unos años antes de su fallecimiento en 1949 y Pérez Temprado en su nostálgico retiro interior, hasta 1954, pudieron vislumbrar el cambio a nuevos tiempos.

17. M. Pallarés, “Aragón y el fuero de Sobrarbe. Estudio histórico documental”, Barcelona, Imprenta de J. Huguet, Sucr. De Inglada y $\mathrm{C}^{\mathrm{a}}, 1917$. 
Matías Pallarés, el primero en fallecer, tan prematuramente a sus cincuenta años, en 1924 en Barcelona, dedicó los tres últimos años, del 20 al 23, a sus investigaciones del Servei del Institut en Cataluña: en 1921 excavaba el recinto de Olérdola, en 1922 descubría las pinturas rupestres de la Cova de Culla, en Benifallet, en cuyo mismo término municipal excavaba la Cova de Almediella y, en colaboración con Luis Pericot (entonces terminando su tesis doctoral, que presentaría al año siguiente), las cuevas del Montgrí; finalmente, sería la excavación de otra cueva, su tarea de campo preferida, en este caso la de Llorá en 1923, su último trabajo arqueológico de campo. La pronta entrega por Pallarés de los informes, permitió incluir el primero, "Excavacions a Olérdola", en el Anuari VI, de 1915-20, y a Bosch anunciar en su "Necrología: Maties Pallarés", en $1925^{18}$, la publicación de los restantes informes en el siguiente Anuari VII, de 1921-26, entonces en preparación: "Cova d'Almediella" y en colaboración con Pericot, "Les coves del Mongrî" y "La cova de Llorá".

Desde la muerte de Pallarés, las actividades de Vidiella y Bardavíu apenas duraron hasta el final de la década, al fallecer ambos en 1929, apenas un lustro después.

Santiago Vidiella, el de más edad del grupo, seguía en estos años en su Calaceite natal y lugar permanente de vida, con su ejercicio de abogado y enfrascado como siempre en la historia regional y, también con prestigio, en el derecho civil aragonés; en 1926 publicaba, en la revista "Universidad" de Zaragoza y con ejemplares de edición separada," Un rector de Valdeltormo. Vida y obras del ilustre bajoaragonés D. Evaristo Colera Soldevilla", biografía ambientada en su contexto regional, basada en los propios manuscritos inéditos del biografiado, prestados para tal fin por su heredero Bardavíu, que documentaba el legado en el prólogo del libro. En estos sus últimos años, Vidiella debió dedicarse intensamente a sus escritos y borradores acumulados, conforme lo evidenciaría su legado, al morir en 1929, en Calaceite, a los sesenta y nueve años de edad ${ }^{19}$.

Vicente Bardavíu, trasladado a Zaragoza, de párroco de San Miguel de los Navarros, desarrollaría desde esa ciudad y durante esta década toda su aportación arqueológica, con excavaciones de una serie de los yacimientos anteriormente controlados, en su primera publicación arqueológica, de 1914, y cuyo interés reiteraba últimamente en 1918: en 1920 y 1922 daba a conocer sendos hallazgos regionales, fuera del ámbito bajoaragonés, un "Informe acerca de los hallazgos prehistóricos de Sena, realizados entonces por R. Gúdel, presbítero beneficiado de la parroquia de dicha localidad oscense, y "Un depósito de hachas de cobre de Egea de los Caballeros" (Zaragoza), a cuyas notas, publicadas en el "Boletín de la Real Academia de Nobles y Bellas Artes de San Luis de Zaragoza", siguió, en el mismo año, su discurso de ingreso en dicha institución, sobre unas supuestas manifestaciones de "El paleolítico inferior de los montes de Torrero: industria, arte y religión de los hombres que en él vivieron", a lo que seguiría, en tono similar aunque conceptualmente menos fantasioso, su publicación de 1923, "Talleres líticos del hombre prehistórico, descubiertos en Alcañiz y en sus entornos", discurso también de recepción en otra academia zaragozana, la de Ciencias Exactas, Físico-Químicas y Naturales, trabajos ambos afortunadamente superados por la inmediata aportación de sus excavaciones de los poblados de Alcañiz, realizadas por Pierre Paris y Bardavíu, asociados en intervenciones de L'École des Hautes Etudes Hispaniques, de Burdeos, sucesivamente: en 1924, el Cabezo del Cuervo; en 1924-25, Tarratrato; en 1927, Cabezo del Moro; y en 1928, Alcañiz el Viejo y El Palao. Los excavados hasta 1926 fueron puntualmente publicados en vida de Bardavíu: Paris y Bardavíu, "Excavaciones en el Cabezo del Cuervo, término de Alcañiz (Teruel)", 1924; Bardavíu, "La estación ibérica de Tarratrato" y "Los poblados ibéricos de Alcañiz en la cuenca del Guadalope y en la del Regallo o Valmuel", 1926; Paris y Bardavíu, "Fuilles dans la region d'Alcañiz (province de Teruel): I, Le Cabezo del Cuervo;

18. P. Bosch Gimpera, "Necrologìa: Maties Pallarés", en "Butlletí de l’Associació Catalana d’Antropología, Etnología i Prehistòria", vol III, fasc.1, Barcelona, 1925, p.110-111.7

19. Todos sus escritos inéditos quedaron ordenados en: a) "Estudios jurídicos aragoneses"(varios trabajos premiados) y "Derechocivil aragonés"(en tres tomos); b) historia regional: "Contribución al catálogo de comendadores de Alcañiz. Orden de Calatrava", "Recopilación de noticias de la historia regional" y "Florilegio de nobles tierra-bajinos"; c) "A puntes misceláneos" (en cuatro tomos, dos de ellos desaparecidos durante la guerra civil) y "Trabajos varios"; y d) "Lecturas y convicciones" (en tres tomos y cuatro apéndices). 
II, Le Tarratrato", 1926. Muerto Bardavíu, a los sesenta y cuatro años, en Zaragoza en 1929, los últimos yacimientos excavados se publicaron un año después, firmados por él y $\mathrm{R}$. Thouvenot, "Fouilles dans la region d’Alcañiz (province de Teruel. I, Alcañiz el Viejo; II, El Palao; III, Cabezo del Moro", 1930. Al año siguiente terminaron estas campañas de L'École des Hautes Etudes Hispaniques, con la excavación parcial, limitada a descubrir únicamente el trazado de la planta del poblado, por $\mathrm{A}$. Bruhl, quien firmaría con P. Paris la memoria aparecida en 1932, "Excavaciones en el Cabezo del Cascarujo, término de Alcañiz".

La necesidad de oficializar las excavaciones arqueológicas era especialmente sentida por Cabré y Pérez Temprado, cuya codirección de la excavación de Azaila, desde 1919 a 1932, y las excavaciones del segundo en poblados de Fabara, Caspe y Chiprana (Zaragoza) y Batea (Tarragona), entre 1923 y 1932, correspondieron todas a los planes de la Junta Superior de Excavaciones y Antigüedades.

En definitiva, desde las intervenciones iniciales de Breuil con Cabré en Cretas y de Pijoan en Calaceite en 1908 , las campañas del Institut dirigidas por Bosch desde 1914 a 1920 y, debordando los años de la década de los veinte, estas actuaciones de Cabré y Pérez Temprado con la Junta Superior , desde 1919 a 1932, y entre los mismos años, las de Paris y Bardavíu con L'Ecole de Burdeos, de 1924 a 1928, y de Bruhl en 1931, deben considerarse como un avance decisivo en la institucionalización de las tareas, hacia una arqueología más rigurosa, científica y socialmente, en consonancia con los procesos culturales de la época.

Cabré y Pérez Temprado alternarían durante estos años las campañas conjuntas de excavación de Azaila con sus restantes actividades personales. Su nombramiento en 1919 como directores de la excavación del Cabezo de Alcalá estuvo precedido por el reconocimiento previo del lugar de las viejas catas de Pablo Gil, solicitado por el Secretario de la Junta, cuyo documento transcribo. "Madrid, 19-7-919/ Junta Superior de Excavaciones y Antigüedades/Sr. D. Lorenzo Pérez Temprado/Muy señor mío, de toda mi consideración: Esta Junta ha designado a V. en unión del Sr. Cabré para practicar excavaciones en Zaida y me permito rogar a V. como conocedor de esas estaciones arqueológicas me indique si es La Zaida o Azaila lo que debe explorarse y donde fueron hallados los vasos que figuran en los Museos de Zaragoza y Barcelona como procedentes de la colección del Sr. Gil. Todos los que han escrito se contradicen. LaZaida aun cuando muy próxima a Azaila y ambas en el río Aguas corresponden uno a Zaragoza y otro a Teruel; la de Zaragoza debe ser del partido judicial de Pina y Azaila del de Híjar. Le ruego me conteste con urgencia para que se dicte la oportuna R. O. de nombramiento y subvención aun cuando por ahora de escasa cantidad. /Aprovecho la ocasión para ofrecerme de V. afectísimo amigo seguro servidor q.l.e.l.m. Francisco Álvarez-Ossrio/ Señas: Secretario de la Junta, Serrano 13, Madrid". Comunicada puntualmente por Pérez Temprado la ubicación del yacimiento, por R.O. de 3 de septiembre del mismo año eran ambos nombrados delegadosdirectores de las excavaciones "de Azaila y Urrea de Gaén". Convertidos los dos amigos en compañeros de trabajo, se organizarían desde entonces para cumplir su responsabilidad compartida de la dirección de las excavaciones durante la década larga de duración de las campañas, hasta 1932; jubilado Pérez Temprado ese mismo año, dejaría también su actividad arqueológica, mientras Cabré reanudaría las excavaciones con dos campañas más, en 1940 y 1942, y sería el autor de toda la bibliografía del yacimiento.

La amistad compartida de ambos se tradujo además en la publicación de las pinturas de Els Secans y del estudio de los materiales del Roquizal, cuyo estudio asumió también Cabré ( Cabré y Pérez Temprado, "Las pinturas rupestres de Els Secans (Mazaleón, Teruel) y sus relaciones etnográficas con la indumentaria actual aragonesa", 1920, y "Nuevos hallazgos de arte rupestre en el Bajo Aragón", 1921;Cabré, "Excavaciones en el Roquizal del Rullo, término de Fabara, provincia de Zaragoza, dirigidas por D. Lorenzo Pérez Temprado", con nota preliminar del excavador del yacimiento, 1929).

Juan Cabré, plenamente integrado en las instituciones centrales españolas, Comisión de Investigaciones Paleontológicas y Prehistóricas, Centro de Estudios Históricos, Museo Cerralbo, convertido de este modo en profesional de la arqueología, armonizaría en estos años la excavación compartida de Azaila con incesantes viajes de inspección y excavaciones oficiales, en trabajos de la Junta Superior, excavando y estudiando 
durante esta década yacimientos del sur, levante y centro de la Península, con publicaciones de la necrópolis de Monachil, pinturas de La Valltorta, Cerro de los Santos, Gabia la Grande, Troya, Las Cogotas, prestando también su atención a algún tema del territorio aragonés, como la publicación del pretendido paleolítico inferior de los alrededores de Zaragoza, y de la propia comarca bajoaragonesa, con las publicaciones citadas de Els Secans del Roquizal y sus entregas sobre las excavaciones de Azaila: Cabré, "Dos tesoros de monedas de bronce autónomas de la necrópolis de Azaila", 1921,'La casa ibérica hasta el tiempo de Augusto, según el plano de la necrópolis ibérica del Cabezo de Alcalá de Azaila",1923, "Los bronces de Azaila", 1925, "Las estilizaciones de caballos y aves de Azaila" y "La cerámica pintada de Azaila", 1926, "Azaila", 1929, "La cerámica pintada de Azaila", 1931; "Un pintor ceramista de Azaila que firmó sus principales obras", 1934-35. Interrumpida la excavación de Azaila en 1932, la reanudaba Cabré en 1940, con sendas campañas, en ese año y en 1942, mientras continuaba con sus publicaciones sobre el yacimiento y sus hallazgos, con un recuerdo también al legado arqueológico de su pueblo natal: Cabré, "La acrópolis de Azaila, Teruel", 1940, "El thymaterium céltico de Calaceite", 1942, "La cerámica céltica de Azaila",1943, "Cerámica de Azaila. Museos Arqueológicos de Madrid, Barcelona y Zaragoza", 1944. Después de tanta actividad, el infatigable Cabré fallecía en Madrid en 1949, con sesenta y nueve años de edad.

Lorenzo Pérez Temprado se había trasladado, de secretario del ayuntamiento, de Mazaleón a Fabara en 1921, dos años después del comienzo de sus excavaciones compartidas con Cabré en Azaila y el mismo de la citada publicación de las pinturas de Els Secans, donde se comunicaba también la existencia del yacimiento cercano de la Botiquería dels Moros, quedando inéditas, de ese mismo término municipal, las pinturas del Salbime, descubiertas en el año anterior a su marcha. Desde Fabara emprendió Pérez Temprado el reconocimiento arqueológico y excavaciones en la zona del interfluvio del Matarraña y Algás, de los términos municipales de Fabara y Maella (Zaragoza) y Batea (Tarragona), y en la zona del Guadalope en su afluencia en el Ebro, del término de Caspe., cuyos resultados, junto al control de algunos yacimientos conocidos y la aportación de nuevas localizaciones, propiciaron la intervención de la Junta Superior de Excavaciones y Antiguiedades, que delegó en Pérez Temprado la dirección de excavaciones en cuatro de los poblados seleccionados, dos de cada zona local: el Roquizal del Rullo, de Fabara, y el Tossal del Moro de Piñeras (Batea, Tarragonza), excavados en sucesivas campañas desde 1923 a 1927, y La Tallada y Palermo, de Caspe, excavados en una primera intervención en 1927 y luego en 1930-31. Se había publicado entonces, por Cabré en 1929, el citado informe sobre los materiales del Roquizal del Rullo, con nota preliminar de su excavador, $y$ apenas tres años después se jubilaba Pérez Temprado de secretario del ayuntamiento, dejando al mismo tiempo su actividad arqueológica, con lo que quedarían inéditas sus excavaciones últimas y una amplia relación de localizaciones, de las que, unos meses antes de su fallecimiento en diciembre de 1954, cumplidos ochenta y nueve años, pudo ver publicadas las pinturas inéditas, Pérez Temprado y Vallespí Pérez, "Las Caídas del Salbime, Mazaleón (Teruel), nuevo yacimiento bajoaragonés con arte rupestre", 1954, y la comunicación de algunos otros de sus controles, en mis primeros trabajos.

Terminaba con ellos el ciclo vital conjunto de su grupo de la generación del "Boletín del Bajo Aragón", que había logrado convertir la comarca en un campo de trabajo arqueológico continuado durante más de treinta años y aceptablemente oficializado. Mediaba entonces el siglo con nuevas perspectivas arqueológicas y el legado que dejaban recibía el reconocimiento académico: desde la Universidad de Zaragoza, A. Beltrán Martínez lo recogía elogiosamente, en 1951, en un balance de urgencia al ocupar la cátedra y desde las universidades de Barcelona y Zaragoza, M. Almagro, A. Beltrán y E. Ripoll le dedicaban en 1956 un estudio monográfico ${ }^{20}$. La Universidad, desde entonces, debía ser garante de la continuidad intergeneracional de la investigación arqueológica.

20. A. Beltrán, "Las investigaciones arqueológicas en Aragón", PSANA 1, Zaragoza, 1951; M. Almagro, A. Beltrán, E. Ripoll, "Prehistoria del Bajo Aragón", Instituto de Estudios Turolenses, 1956. 